\title{
Establishing a Cohort of Transgender Men and Gender Nonconforming Individuals to Understand the Molecular Impact of Testosterone on Breast Physiology
}

\author{
Gabrielle M. Baker, ${ }^{1}$ Michael E. Pyle, ${ }^{1}$ Adam M. Tobias, ${ }^{2}$ Richard A. Bartlett, ${ }^{2}$ Jordana Phillips, ${ }^{3}$
}

Valerie J. Fein-Zachary, ${ }^{3}$ Gerburg M. Wulf, and Yujing J. Heng ${ }^{1, *}$

\begin{abstract}
Purpose: To characterize a cohort of transgender men and masculine-centered gender nonconforming individuals who underwent gender-affirming chest-contouring surgeries at our institution between 2013 and 2018.

Methods: Demographics, medical history, and breast histopathological assessment for 340 patients were retrieved from medical records.

Results: Most of our patients were white, non-Hispanic (75.0\%), were taking testosterone (83.2\%), and opted for chest-contouring surgery after 12-14 months of testosterone therapy. Ten patients were parous (2.9\%). Seventynine (23.2\%) and 27 (7.9\%) patients had a family history of breast cancer or ovarian cancer, respectively. One transgender man was incidentally diagnosed with ductal carcinoma in situ at chest-contouring surgery.

Conclusion: Future studies on this cohort will provide valuable insights about the impact of testosterone on breast physiology.
\end{abstract}

Keywords: breast; gender dysphoria; plastic surgery; top surgery

\section{Background}

The proportion of transgender adults in the United States has increased for the past decade. ${ }^{1}$ With increasing cultural and social acceptance, and insurance access, more transgender individuals are undergoing gender-affirming hormone therapy and/or surgery to alleviate their gender dysphoria. Specifically, transgender men (TM; trans men or female-to-male) and masculine-centered gender nonconforming individuals (GNCIs) are natal females who pursue testosterone therapy and/or gender-affirming surgery to enhance masculinization. ${ }^{2}$ Gender-affirming surgery for TM and GNCIs may include chest contouring, hysterectomy with or without bilateral salpingo-oophorectomy, creation of male genital prostheses, and facial esthetic procedures. ${ }^{3}$ The surgical removal of breast tissue is the most common first surgery for TM and GNCIs. ${ }^{4}$ Unlike mas- tectomy for oncological or breast cancer risk-reducing purposes, chest-contouring surgery in TM and GNCIs emphasizes an esthetic outcome and does not necessarily remove all mammary tissue. ${ }^{5}$

Testosterone therapy is a long-term process and its regimen follows the principle of hormone replacement therapy for cisgender men. The physiological testosterone range for cisgender men is 10 -fold higher than cisgender women $(300-1000 \mathrm{ng} / \mathrm{dL} \text { vs. } 30-100 \mathrm{ng} / \mathrm{dL})^{6}$ Thus, TM and GNCIs use high-dose injectable testosterone esters (50-200 mg/week), transdermal testosterone gel $(2.5-10 \mathrm{~g} /$ day $)$, or transdermal testosterone patch $(2.5-7.5 \mathrm{mg} /$ day) to achieve testosterone levels comparable with cisgender men. ${ }^{6}$ These testosterone doses are $>10$-fold higher than the recommended treatment for hypoactive sexual desire disorder in cisgender women $(300 \mu \mathrm{g} /$ day $) .^{7}$ In other words, the amount of

\footnotetext{
Departments of ${ }^{1}$ Pathology and ${ }^{2}$ Plastic Surgery, Beth Israel Deaconess Medical Center, Harvard Medical School, Boston, Massachusetts.

${ }^{3}$ Division of Breast Imaging, Department of Radiology, Beth Israel Deaconess Medical Center, Harvard Medical School, Boston, Massachusetts.

${ }^{4}$ Division of Hematology/Oncology, Department of Medicine, Beth Israel Deaconess Medical Center, Harvard Medical School, Boston, Massachusetts.

*Address correspondence to: Yujing J. Heng, PhD, Department of Pathology, Beth Israel Deaconess Medical Center, Harvard Medical School, 330 Brookline Avenue, Dana 517B, Boston, MA 02215, E-mail: yheng@bidmc.harvard.edu
}

(C) Gabrielle M. Baker et al. 2019; Published by Mary Ann Liebert, Inc. This Open Access article is distributed under the terms of the Creative Commons License (http://creativecommons.org/licenses/by/4.0), which permits unrestricted use, distribution, and reproduction in any medium, provided the original work is properly cited. 
exogenous testosterone taken by TM and GNCIs is significantly higher than what is typically taken by cisgender women. Such long-term high-dose exposure to exogenous testosterone in TM and GNCIs raises concerns about the effect of testosterone therapy on their health.

Although testosterone therapy is generally considered safe, ${ }^{8}$ data are limited and there remains a knowledge gap about the impact of exogenous testosterone on hormone-sensitive tissues and hormone-dependent malignancies, for example, the breast and breast cancer. Surgical breast specimens from TM and GNCIs are a valuable resource to better understand the mechanism of action of long-term high-dose testosterone on breast physiology. This report describes our Transgender and Testosterone Therapy use (i.e., "Triple T") research cohort consisting of TM and GNCIs with available archival breast tissue and histopathological slides at our institution between 2013 and 2018.

\section{Methods}

\section{Subjects}

This retrospective study was approved by the Beth Israel Deaconess Medical Center (BIDMC) Committee for Clinical Investigations (Protocol Number: 2018P000814). A total of 340 patients with gender dysphoria who underwent chest-contouring surgery between January 2013 and December 2018 at BIDMC, Boston, MA, were identified using surgical case lists from two plastic surgeons who perform the vast majority of chest-contouring surgeries at our institution.

\section{Data collection}

Patient data from presurgical consult questionnaires, referral letters, and postsurgery follow-up were retrieved through electronic medical records. The electronic medical records were accessed between February 2019 and June 2019. Presurgical data include self-identified gender, home state, race/ethnicity, accompanying support person during consult, length and type of testosterone therapy, hormonal birth control use, body mass index (BMI), family history of breast and ovarian cancer, medical history including hysterectomy, reproductive history, smoking status, and alcohol consumption. Postsurgery data include age at surgery, breast histopathological findings, and updated information about hysterectomy and/or bilateral salpingo-oophorectomy. Length of testosterone use at surgery accounts for the additional time between presurgical consult and date of surgery, and reflects the duration of breast tissue exposed to testosterone.

\section{Analysis}

Comparisons between two groups of patients who did and did not take testosterone therapy were analyzed using Mann-Whitney $U$ test for continuous variables and Fisher's test for categorical variables. All analyses were conducted using $\mathrm{R}$ version 3.4.0 and $p<0.05$ was considered statistically significant.

\section{Results}

Table 1 displays the demographics of our Triple $\mathrm{T}$ research cohort. The number of chest-contouring surgeries at BIDMC increased from 1 to 112 cases per year between 2013 and 2018. Our geographically diverse patients were from 15 U.S. states (CO, CT, MA, ME, NC, $\mathrm{NH}, \mathrm{NJ}, \mathrm{NY}, \mathrm{OH}, \mathrm{PA}, \mathrm{RI}, \mathrm{TN}, \mathrm{TX}, \mathrm{VA}$, and VT) and $83.8 \%$ of them reside in MA. Most of our patients identified as TM (77.9\%), $10.9 \%$ identified as GNCI, and $11.2 \%$ did not self-identify. The vast majority of our patients were white, non-Hispanic (75.0\%). Thirty-six patients $(10.6 \%)$ had hysterectomy and/or bilateral salpingo-oophorectomy after chest-contouring surgery

\section{Table 1. Demographics of the 340 Transgender Men and Gender Nonconforming Individuals Who Had Chest-Contouring Surgeries at Beth Israel Deaconess Medical Center Between January 2013 and December 2018}

\begin{tabular}{lc}
\hline & $n(\%)$ \\
\hline Year of surgery & \\
2013 & $1(0.3)$ \\
2014 & $7(2.1)$ \\
2015 & $40(11.8)$ \\
2016 & $75(22.1)$ \\
2017 & $105(30.9)$ \\
2018 & $112(32.9)$ \\
Gender as identified by patient & \\
Trans male & $265(77.9)$ \\
Nonconforming & $37(10.9)$ \\
Not specified & $38(11.2)$ \\
Race and ethnicity & \\
White, non-Hispanic & $255(75.0)$ \\
White, Hispanic & $7(2.1)$ \\
Black or African American, non-Hispanic & $28(8.2)$ \\
Black or African American, Hispanic & $2(0.6)$ \\
Asian & $11(3.2)$ \\
Native American/Pacific Islander & $3(0.9)$ \\
Mixed race, non-Hispanic & $8(2.4)$ \\
Unspecified race and ethnicity & $26(7.6)$ \\
Companion at presurgery consult & \\
One or both parents & $25(7.4)$ \\
Other family member & $3(0.9)$ \\
Romantic partner & $42(12.4)$ \\
Spouse & $3(0.9)$ \\
Friend & $4(1.2)$ \\
Unknown & $263(77.4)$ \\
Hysterectomy and/or salpingo-oophorectomy & \\
Yes, before chest contouring & $26(7.6)$ \\
Yes, after chest contouring & $36(10.6)$ \\
No & $278(81.8)$ \\
\hline
\end{tabular}


compared with 26 (7.6\%) who had the same procedure before chest-contouring surgery; 278 (81.8\%) had not pursued this procedure at the time of medical record review. One patient had a previous chest wall reconstruction and six had previous breast reduction surgeries.

Among 340 patients, 283 (83.2\%) reported taking testosterone therapy before chest-contouring surgery compared with 55 (16.2\%) who did not (Table 2). Testosterone use was not recorded for 2 patients $(0.6 \%)$. The most frequent testosterone formulation was intra- muscular testosterone enanthate/cypionate $(249 / 283$; $88.0 \%)$, followed by transdermal gel $(14 / 283 ; 4.9 \%)$, transdermal patch $(5 / 283 ; 1.8 \%)$, subcutaneous pellet $(3 / 283 ; 1.1 \%)$, and transdermal cream $(1 / 283 ; 0.4 \%)$. Testosterone formulation for 11 patients was unknown (3.9\%). Most patients (38.9\%) opt for chest-contouring surgery after pursuing testosterone therapy between 12 and 24 months (Table 2).

Of the available nonmodifiable breast cancer risk factors, $10(2.9 \%)$ of our patients reported parity, 79

Table 2. Clinical Characteristics and Breast Cancer Risk Factors of the 340 Patients, Stratified by Testosterone Therapy Use

\begin{tabular}{|c|c|c|c|c|}
\hline & All patients & Testosterone use & No testosterone use & $p^{*}$ \\
\hline$n(\%)$ & $340(100.0)$ & $283(83.2)$ & $55(16.2)$ & \\
\hline Age at surgery (median [IQR]) & $25.5[22.0,30.0]$ & $25.0[21.0,30.0]$ & $28.0[24.0,32.5]$ & 0.09 \\
\hline \multicolumn{4}{|c|}{ Length of testosterone therapy at the time of chest-contouring surgery, $n(\%)$} & - \\
\hline$<1$ year & $62(18.2)$ & $62(21.9)$ & - & \\
\hline$\geq 1$ to $<2$ years & $110(32.4)$ & $110(38.9)$ & - & \\
\hline$\geq 2$ to $<5$ years & $66(19.4)$ & $66(23.3)$ & - & \\
\hline$\geq 5$ years & $14(4.1)$ & $14(4.9)$ & - & \\
\hline Current user, unknown duration & $31(9.1)$ & $31(11.0)$ & - & \\
\hline Never use & $55(16.2)$ & - & $55(100.0)$ & \\
\hline Unknown & $2(0.6)$ & - & - & \\
\hline \multicolumn{5}{|l|}{ Reproductive and hormonal factors } \\
\hline Age of menarche (median [IQR]) & $12.5[11.0,13.0]$ & $12.0[11.0,13.0]$ & $13.0[12.0,13.5]$ & 0.97 \\
\hline Parity, $n(\%)$ & & & & 0.46 \\
\hline Have children & $10(2.9)$ & $7(2.5)$ & $3(5.5)$ & \\
\hline No children & $148(43.5)$ & $124(43.8)$ & $24(43.6)$ & \\
\hline Unknown & $182(53.5)$ & $152(53.7)$ & $28(50.9)$ & \\
\hline \multicolumn{4}{|l|}{ Hormone birth control use, $n(\%)$} & $<0.01$ \\
\hline Yes & $16(4.7)$ & $8(2.8)$ & $8(14.5)$ & \\
\hline No & $324(95.3)$ & $275(97.2)$ & $47(85.5)$ & \\
\hline \multicolumn{5}{|l|}{ Previous benign breast disease, $n$ (\%) } \\
\hline Yes & $12(3.5)$ & $9(3.2)$ & $3(5.5)$ & 0.42 \\
\hline No & $328(96.5)$ & $274(96.8)$ & $52(94.5)$ & \\
\hline \multicolumn{5}{|l|}{ Family history } \\
\hline Family history of breast cancer, $n(\%)$ & & & & 0.22 \\
\hline Yes, first degree & $8(2.4)$ & $6(2.1)$ & $2(3.6)$ & \\
\hline Yes, second degree & 47 (13.8) & $41(14.5)$ & $6(10.9)$ & \\
\hline Yes, unknown degree & $24(7.1)$ & $20(7.1)$ & $3(5.5)$ & \\
\hline No & $202(59.4)$ & $173(61.1)$ & $29(52.7)$ & \\
\hline Unknown & 59 (17.4) & $43(15.2)$ & $15(27.3)$ & \\
\hline \multicolumn{4}{|l|}{ Family history of ovarian cancer, $n(\%)$} & 0.16 \\
\hline Yes & $27(7.9)$ & $24(8.5)$ & $3(5.5)$ & \\
\hline No & $234(68.8)$ & $200(70.7)$ & $34(61.8)$ & \\
\hline Unknown & $79(23.2)$ & $59(20.8)$ & $18(32.7)$ & \\
\hline \multicolumn{5}{|l|}{ Modifiable risk factors } \\
\hline BMI at surgery (median [IQR]) & $26.1[23.5,30.4]$ & $26.4[23.7,30.5]$ & $25.1[22.0,29.6]$ & 0.54 \\
\hline \multicolumn{4}{|l|}{ Alcohol consumption, $n(\%)$} & 0.03 \\
\hline Current & $188(55.3)$ & $148(52.3)$ & 39 (70.9) & \\
\hline Never & $142(41.8)$ & $126(44.5)$ & $16(29.1)$ & \\
\hline Unknown & $10(2.9)$ & $9(3.2)$ & $0(0.0)$ & \\
\hline \multicolumn{4}{|l|}{ Tobacco smoker, $n$ (\%) } & 0.72 \\
\hline Current & $23(6.8)$ & $21(7.4)$ & $2(3.6)$ & \\
\hline Ever & $32(9.4)$ & $25(8.8)$ & $6(10.9)$ & \\
\hline Never & $281(82.6)$ & $233(82.3)$ & $47(85.5)$ & \\
\hline Unknown & $4(1.2)$ & $4(1.4)$ & $0(0.0)$ & \\
\hline
\end{tabular}

${ }^{*} p$ values were obtained using Mann-Whitney $U$ or Fisher's test to compare between patients who did and did not use testosterone. Bold indicates $p$ values that are $<0.05$.

$\mathrm{BMI}$, body mass index; IQR, interquartile range. 
(23.2\%) indicated a family history of breast cancer and $27(7.9 \%)$ indicated a family history of ovarian cancer (Table 2). Twelve (3.5\%) had prior benign breast disease: four were fibroadenomas, one was a cyst, and the histological subtype of the other seven cases were not recorded. Most of our patients did not use hormonal birth control $(95.3 \%)$. The majority of them consumed alcohol (55.3\%; Table 2). The average BMI of our Triple $\mathrm{T}$ cohort was 26.1 .

Detailed histopathological review of surgical specimens of a subset of 115 patients have been conducted and reported elsewhere. ${ }^{9}$ Four of these 115 patients had atypical hyperplasia: 2 had atypical ductal hyperplasia $(\mathrm{ADH}), 1$ had atypical lobular hyperplasia (ALH), and 1 had both ADH and ALH. Atypical hyperplasia is a proliferative histological subtype of benign breast disease that is known to increase subsequent breast cancer risk. ${ }^{10,11}$ One TM, 29 years old, was incidentally diagnosed with ductal carcinoma in situ at chest-contouring surgery. They had been taking testosterone cypionate $80 \mathrm{mg} /$ week for 4 years, and their breast cancer 23-genes screening panel was negative, including negative for $B R C A$ mutation. ${ }^{5}$

\section{Discussion}

As the number of transgender adults increases, more research is critically needed to understand the impact of gender-affirming hormonal therapy and/or surgery on the health outcomes in this historically discriminated population. The impact of exogenous testosterone on hormone-dependent malignancies, such as breast cancer, in these individuals remains unclear. ${ }^{12}$ In this report, we described our Triple T cohort that consisted of $340 \mathrm{TM}$ and GNCIs who had chest-contouring surgeries at our institution from 2013 to 2018 . We collected clinical data, including testosterone therapy details, archival breast tissue blocks, and histopathology slides, to design future tissue-based studies to elucidate the molecular influence of testosterone on breast physiology and breast cancer risk in TM and GNCIs. Our future study will be valuable in supporting the health management of TM and GNCIs who pursue testosterone therapy.

Three population-based studies concluded that breast cancer risk in TM is lower than cisgender women. ${ }^{13-15}$ However, these studies only reported a limited number of 12 cases of breast cancer among 3698 TM and none of these studies characterized the genetic risk of those individuals. We categorized several important factors related to breast cancer risk in our cohort: $\mathrm{BMI},{ }^{16}$ reproductive factors, ${ }^{16}$ family history, ${ }^{17}$ previous benign breast disease, ${ }^{18}$ alcohol consumption, ${ }^{19}$ and tobacco smoking. ${ }^{20}$ It is important to note that these factors are associated with increased breast cancer risk in cisgender women. Breast cancer risk factors for TM and GNCIs have yet to be established. Based on our limited cohort numbers, these breast cancer risk factors for cisgender women do not appear to be different between TM and GNCIs who do and do not take testosterone therapy. More long-term follow-up studies are needed to identify factors that modulate breast cancer risks unique to TM and GNCIs. The histopathological findings of atypical hyperplasia and DCIS in a subset of our surgical specimens reiterates the need to establish culturally sensitive breast cancer screening protocols for TM and GNCIs, especially for those who do not undergo chest-contouring surgery.

The interplay between endogenous estrogens and exogenous testosterone in the breast remains poorly understood. ${ }^{21}$ Under conditions of excess, testosterone can be partially aromatized to estradiol and may drive breast cancer development. ${ }^{22}$ Indeed, higher plasma testosterone levels are associated with increased breast cancer risk in both pre- and postmenopausal cisgender women. ${ }^{23,24}$ Bentz et al. conducted the first and only molecular study in TM with five paired breast biopsies taken before and after 2 years of testosterone therapy. ${ }^{25}$ They reported that testosterone therapy was associated with a breast cancer gene signature and testosterone may initiate breast carcinogenesis. ${ }^{25}$ Therefore, larger observational studies are critically needed to assess how testosterone affects breast tissue health in TM and GNCIs. Neither testosterone use nor minimum length of testosterone use is mandatory for chest-contouring surgery. Thus, patients who did not use testosterone are ideal controls for subsequent research studies.

One challenge in conducting research to understand the molecular influence of testosterone on breast tissues is the unknown cumulative testosterone exposure and the lack of biomarkers to approximate androgen levels in the breast. Throughout the duration of therapy, an individual can interchange testosterone formulations (due to costs, availability, or personal preference), adjust testosterone dosage to optimize well-being, or pause therapy to conceive. Optimal testosterone dosing is not determined solely by serum levels but also by the patient's desire and whether their desired masculinizing effect is achieved. No study has systematically compared testosterone serum levels and the levels of testosterone and $5 \alpha$-dihydrotestosterone in breast tissues. One potential biomarker could be the evaluation of androgen 
receptor expression as a surrogate to reflect androgen levels in the breast. ${ }^{26-28}$

The implementation of Section 1557 of the Affordable Care Act (ACA), signed into law in May 2016, ${ }^{29}$ partially explains the rapid increase in the number of chest-contouring surgeries conducted at BIDMC in 2017 and 2018. As expected, most of our patients underwent chest-contouring surgery before hysterectomy and/or bilateral salpingo-oophorectomy. ${ }^{4}$ With increasing cultural and social acceptance, and steadily improving health insurance policy coverage (i.e., access to better health care), ${ }^{30}$ we expect more TM and GNCIs to pursue chest-contouring surgeries at BIDMC, thus substantially increasing the numbers of our Triple $\mathrm{T}$ cohort in the next few years. As our cohort grows, we will continuously improve and update our questionnaires to capture valuable patient data, for example, how long they have been using a specific testosterone formulation. We could also perform blood work at time of presurgical workup, and collect fresh frozen breast tissues at the time of surgery.

\section{Author Disclosure Statement}

No competing financial interests exist.

\section{Funding Information}

Y.J.H. is supported by the Klarman Family Foundation.

\section{References}

1. Meerwijk EL, Sevelius JM. Transgender population size in the United States: a meta-regression of population-based probability samples. Am J Public Health. 2017;107:e1-e8.

2. Hembree WC, Cohen-Kettenis PT, Gooren L, et al. Endocrine treatment of gender-dysphoric/gender-incongruent persons: an endocrine society clinical practice guideline. J Clin Endocrinol Metab. 2017;102:3869-3903.

3. Coleman E, Bockting W, Botzer M, et al. Standards of care for the health of transsexual, transgender, and gender-nonconforming people, version 7. Int J Transgenderism. 2012;13:165-232.

4. Frederick MJ, Berhanu $A E$, Bartlett R. Chest surgery in female to male transgender individuals. Ann Plast Surg. 2017;78:249-253.

5. Eismann J, Heng YJ, Fleischmann-Rose K, et al. Interdisciplinary management of transgender individuals at risk for breast cancer: case reports and review of the literature. Clin Breast Cancer. 2019;19:e12-e19.

6. Gardner IH, Safer JD. Progress on the road to better medical care for transgender patients. Curr Opin Endocrinol Diabetes Obes. 2013;20:553-558.

7. Braunstein GD, Sundwall DA, Katz M, et al. Safety and efficacy of a testosterone patch for the treatment of hypoactive sexual desire disorder in surgically menopausal women. Arch Intern Med. 2005;165:1582.

8. T'Sjoen G, Arcelus J, Gooren L, et al. Endocrinology of transgender medicine. Endocr Rev. 2018;40:97-117.

9. Torous VF, Schnitt SJ. Histopathologic findings in breast surgical specimens from patients undergoing female-to-male gender reassignment surgery. Mod Pathol. 2019;32:346-353.

10. Menes TS, Kerlikowske K, Lange J, et al. Subsequent breast cancer risk following diagnosis of atypical ductal hyperplasia on needle biopsy. JAMA Oncol. 2017;3:36-41.

11. Dupont WD, Page DL. Risk factors for breast cancer in women with proliferative breast disease. N Engl J Med. 1985;312:146-151.

12. Joint $\mathrm{R}$, Chen $\mathrm{ZE}$, Cameron $\mathrm{S}$. Breast and reproductive cancers in the transgender population: a systematic review. BJOG. 2018;125:1505-1512.

13. Brown GR, Jones KT. Incidence of breast cancer in a cohort of 5,135 transgender veterans. Breast Cancer Res Treat. 2015;149:191-198.
14. De Blok CJM, Wiepjes CM, Nota NM, et al. Breast cancer risk in transgender people receiving hormone treatment: nationwide cohort study in the Netherlands. BMJ. 2019;365:11652.

15. Gooren $\sqcup$, van Trotsenburg MAA, Giltay EJ, van Diest PJ. Breast cancer development in transsexual subjects receiving cross-sex hormone treatment. J Sex Med. 2013;10:3129-3134.

16. Collaborative Group on Hormonal Factors in Breast Cancer. Menarche, menopause, and breast cancer risk: individual participant meta-analysis, including 118964 women with breast cancer from 117 epidemiological studies. Lancet Oncol. 2012;13:1141-1151.

17. Colditz GA, Rosner B. Cumulative risk of breast cancer to age 70 years according to risk factor status: data from the nurses' health study. Am J Epidemiol. 2000;152:950-964.

18. Dyrstad SW, Yan Y, Fowler AM, Colditz GA. Breast cancer risk associated with benign breast disease: systematic review and meta-analysis. Breast Cancer Res Treat. 2015;149:569-575.

19. Hirko KA, Chen WY, Willett WC, et al. Alcohol consumption and risk of breast cancer by molecular subtype: prospective analysis of the nurses' health study after 26 years of follow-up. Int J Cancer. 2016;138:1094-1101.

20. Gaudet MM, Carter BD, Brinton LA, et al. Pooled analysis of active cigarette smoking and invasive breast cancer risk in 14 cohort studies. Int $J$ Epidemiol. 2017;46:881-893.

21. Dimitrakakis C. Androgens and breast cancer in men and women Endocrinol Metab Clin North Am. 2011;40:533-547.

22. Shao T, Grossbard ML, Klein P. Breast cancer in female-to-male transsexuals: two cases with a review of physiology and management. Clin Breast Cancer. 2011;11:417-419.

23. Kaaks R, Tikk K, Sookthai D, et al. Premenopausal serum sex hormone levels in relation to breast cancer risk, overall and by hormone receptor status-results from the EPIC cohort. Int J Cancer. 2014;134:1947-1957.

24. Kaaks R, Rinaldi S, Key TJ, et al. Postmenopausal serum androgens, oestrogens and breast cancer risk: the European prospective investigation into cancer and nutrition. Endocr Relat Cancer. 2005;12:1071-1082.

25. Bentz EK, Pils D, Bilban M, et al. Gene expression signatures of breast tissue before and after cross-sex hormone therapy in female-to-male transsexuals. Fertil Steril. 2010;94:2688-2696.

26. Kensler KH, Regan MM, Heng YJ, et al. Prognostic and predictive value of androgen receptor expression in postmenopausal women with estrogen receptor-positive breast cancer: results from the Breast International Group Trial 1-98. Breast Cancer Res. 2019;21:30.

27. Kensler $\mathrm{KH}$, Beca F, Baker GM, et al. Androgen receptor expression in normal breast tissue and subsequent breast cancer risk. NPJ Breast Cancer. 2018:4:33.

28. Kensler $\mathrm{KH}$, Poole EM, Heng YJ, et al. Androgen receptor expression and breast cancer survival: results from the nurses' health studies. JNCI J Natl Cancer Inst. 2019 Nov 15;In Press. PMCID: PMC6624168.

29. Office of Civil Rights. Summary: Final Rule Implementing Section 1557 of the Affordable Care Act. US Department of Health and Civil Rights, 2017.

30. Dowshen NL, Christensen J, Gruschow SM. Health insurance coverage of recommended gender-affirming health care services for transgender youth: shopping online for coverage information. Transgender Health. 2019;4:131-135.

Cite this article as: Baker GM, Pyle ME, Tobias AM, Bartlett RA, Phillips J, Fein-Zachary VJ, Wulf GM, Heng YJ (2019) Establishing a cohort of transgender men and gender nonconforming individuals to understand the molecular impact of testosterone on breast physiology, Transgender Health 4:1, 326-330, DOI: 10.1089/trgh.2019.0040.

$\begin{aligned} & \text { Abbreviations Used } \\ & \mathrm{ADH}=\text { atypical ductal hyperplasia } \\ & \mathrm{ALH}=\text { atypical lobular hyperplasia } \\ & \mathrm{BIDC}=\text { Beth Israel Deaconess Medical Center } \\ & \mathrm{BMI}=\text { body mass index } \\ & \mathrm{GNCl}=\text { gender nonconforming individual } \\ & \mathrm{TM}=\text { transgender men }\end{aligned}$

